\title{
QUOTIENT RINGS OF ENDOMORPHISM RINGS OF MODULES WITH ZERO SINGULAR SUBMODULE
}

\author{
JOHN HUTCHINSON AND JULIUS ZELMANOWITZ
}

\begin{abstract}
Throughout this paper $(R, M, N, S)$ will denote a Morita context satisfying a certain nonsingularity condition. For such contexts we give necessary and sufficient conditions in terms of $M$ and $R$ for $S$ to have a semisimple maximal left quotient ring; respectively a full linear maximal left quotient ring, a semisimple classical left quotient ring. In doing so we extend the corresponding well-known theorems for rings (employing them in the process) to endomorphism rings.
\end{abstract}

Suppose $(R, M, N, S)$ is a Morita context ([1], [2]). That is suppose ${ }_{R} M_{S}$ and ${ }_{S} N_{R}$ are bimodules with an $R$ - $R$ bimodule homomorphism ( , ): $M \otimes_{S} N \rightarrow R$ and an $S$-S bimodule homomorphism [, ]:N $\otimes_{R} M \rightarrow S$ satisfying

$$
m_{1}\left[n_{1}, m_{2}\right]=\left(m_{1}, n_{1}\right) m_{2} \quad \text { and } \quad n_{1}\left(m_{1}, n_{2}\right)=\left[n_{1}, m_{1}\right] n_{2}
$$

for all $m_{1}, m_{2} \in N$ and $n_{1}, n_{2} \in N$.

Throughout, unless otherwise indicated, $M$ and $N$ will satisfy the following condition: $M_{S}$ is faithful; and $[N, m]=0$ for $m \in M$ implies that $m=0$.

Note that when this condition is satisfied, we can (and will) assume that $S \subseteq \operatorname{Hom}_{R}(M, M)$.

Let ${ }_{R} M$ be any left $R$-module, and set $N=\operatorname{Hom}_{R}(M, R)$ and $S=$ $\operatorname{Hom}_{R}(M, M)$. Set $(m, f)=(m) f$ for $m \in M, f \in N$; and $[f, m]$ is defined via $m_{1}[f, m]=\left(m_{1}, f\right) m$ for all $m, m_{1} \in M, f \in N$. Then $(R, M, N, S)$ is a Morita context, called the standard context for ${ }_{R} M$.

If $R$ is semiprime and ${ }_{R} M$ is torsionless, then the above condition is satisfied by the standard Morita context for ${ }_{R} M$. If ${ }_{R} M$ is a generator and $1 \in R$; or indeed, if (Trace $\left.{ }_{R} M\right) m \neq 0$ whenever $0 \neq m \in M$, then the standard Morita context for ${ }_{R} M$ satisfies the above condition.

Lemma 1. (a) If $A$ is an essential left ideal of $S$, then $M A$ is an essential submodule of ${ }_{R} M$.

Received by the editors November 8,1971 .

AMS 1970 subject classifications. Primary 16A08, 16A64; Secondary 16A12, 16A42.

Key words and phrases. Endomorphism rings, Morita contexts, singular submodule, quotient rings, full linear rings, Goldie dimension.

(c) American Mathematical Society 1972 
(b) If $K$ is an essential submodule of ${ }_{R} M$, then

$$
[N, K]=\left\{\sum_{i=1}^{t}\left[n_{i}, k_{i}\right] \mid n_{i} \in N, k_{i} \in K\right\}
$$

is an essential left ideal of $S$.

Proof. (a) If $0 \neq m \in M$, then $[N, m] \cap A \neq 0$. Since $M_{S}$ is faithful, $0 \neq M([N, m] \cap A) \subseteq M[N, m] \cap M A=(M, N) m \cap M A \subseteq R m \cap M A$.

(b) If $0 \neq s \in S$, then $M s \cap K \neq 0$. Hence $0 \neq[N, M s \cap K] \subseteq[N, M s] \cap$ $[N, K] \subseteq S s \cap[N, K]$.

Proposition 2. $Z\left({ }_{R} M\right)=0$ if and only if $Z\left({ }_{S} S\right)=0$.

Proof. Suppose $Z\left({ }_{R} M\right)=0$, and let $s \in Z\left({ }_{S} S\right)$. Then $A s=0$ where $A$ is an essential left ideal of $S$. By the preceding lemma, $M A$ is an essential submodule of ${ }_{R} M$, and clearly $(M A) s=0$. Hence $\operatorname{ker} s$ is essential in ${ }_{R} M$, and since $Z\left({ }_{R} M\right)=0$ it follows that $s=0$.

Conversely suppose that $Z\left({ }_{R} M\right) \neq 0$. If $0 \neq z \in Z\left({ }_{R} M\right)$ it follows from the condition on [, ] that there is an $n \in N$ with $0 \neq[n, z]$. Set $s=[n, z]$. We claim that $s \in Z\left({ }_{S} S\right)$, and by the previous lemma it suffices to prove that ker $s$ is essential in ${ }_{R} M$. If $m \in M, m \notin \operatorname{ker} s$, then $(m, n) \neq 0$. Since $z \in Z\left({ }_{R} M\right)$ there exists $a \in R^{1}$ ( $R^{1}$ denotes $R$ with identity adjoined in the customary manner) with $a(m, n) \neq 0$ and $a(m, n) z=0$. But then ams= $a m[n, z]=a(m, n) z=0$. Hence $0 \neq a m \in \operatorname{ker} s$ and ker $s$ is essential in ${ }_{R} M$.

Proposition 3 [1, p. 276]. $d\left({ }_{R} M\right)=d\left({ }_{S} S\right)$.

Proof. Here $d\left({ }_{R} M\right)$ denotes the (Goldie) dimension of ${ }_{R} M$. If $\oplus_{i} A_{i}$ is an internal direct sum of nonzero left ideals of $S$, then a routine calculation shows that $\sum_{i} M A_{i}$ is a direct sum of nonzero submodules of ${ }_{R} M$. Hence $d\left({ }_{S} S\right) \leqq d\left({ }_{R} M\right)$. On the other hand if $\oplus_{i} M_{i}$ is an internal direct sum of nonzero submodules of ${ }_{R} M$ and $A_{i}=\left[N, M_{i}\right]$, then $\sum_{i} A_{i}$ is a direct sum of nonzero left ideals of $S$. Hence $d\left({ }_{R} M\right) \leqq d\left({ }_{S} S\right)$.

Let $\Lambda=\operatorname{Hom}_{R}(M, M)$ and $\Omega=\operatorname{Hom}_{R}(\bar{M}, \bar{M})$ where $\bar{M}$ is the injective hull of ${ }_{R} M$. As we have already noted, we can assume that $S \subseteq \Lambda$. When $Z\left({ }_{R} M\right)=0, \Omega$ is a regular self-injective ring [5] and we can assume that $\Lambda \subseteq \Omega$.

Proposition 4. When $Z\left({ }_{k} M\right)=0, \Omega$ is the maximal left quotient ring of $S$.

Proof. Given $0 \neq(1) \in \Omega, M_{(1)^{-1} \cap M}$ is an essential submodule of ${ }_{R} M$, and so $\left(M(1)^{-1} \cap M\right)(1) \neq 0$. Hence

$$
0 \neq\left[N,\left(M_{(1)^{-1}}^{-1} \cap M\right)(1)\right]=\left[N, M_{(1)^{-1}} \cap M\right](1) \subseteq S \cap S(1) .
$$


Since $Z\left({ }_{S} S\right)=0$ it follows that $\Omega$ is a maximal left quotient ring of $S$.

THEOREM 5. S has a semisimple maximal left quotient ring (necessarily isomorphic to $\Omega)$ if and only if $Z\left({ }_{R} M\right)=0$ and $d\left({ }_{R} M\right)<\infty$.

Proof. By [8, Theorem 1.6], $S$ has a semisimple maximal left quotient ring if and only if $Z\left({ }_{S} S\right)=0$ and $d\left({ }_{S} S\right)<\infty$. The theorem then follows from the previous three propositions.

A submodule $K$ of ${ }_{R} M$ is closed if $K$ has no proper essential extensions in ${ }_{R} M$. Let $\mathscr{C}\left({ }_{R} M\right)$ denote the set of closed submodules of ${ }_{R} M$. If $Z\left({ }_{R} M\right)=$ 0 it is well known that $\mathscr{C}\left({ }_{R} M\right)$ is a complete complemented lattice and $\mathscr{C}\left({ }_{R} M\right)$ is lattice isomorphic to $\mathscr{C}\left({ }_{R} \bar{M}\right)$ under contraction [3, p. 61].

Proposition 6. For any module ${ }_{R} M$ (not necessarily satisfying the standing hypothesis) with $Z\left({ }_{R} M\right)=0$ :

(a) If $A \in \mathscr{C}\left({ }_{\Omega} \Omega\right)$, then $\bar{M} A \in \mathscr{C}\left({ }_{R} \bar{M}\right)$.

(b) If $K \in \mathscr{C}\left({ }_{R} \bar{M}\right)$, then $\operatorname{Hom}_{R}(\bar{M}, K) \in \mathscr{C}\left({ }_{\Omega} \Omega\right)$.

(c) $\mathscr{C}\left({ }_{R} \bar{M}\right)$ is lattice isomorphic to $\mathscr{C}\left({ }_{\Omega} \Omega\right)$.

Proof. (a) Since $\Omega$ is regular left self-injective $A=\Omega \varepsilon$ where $\varepsilon^{2}=$ $\varepsilon \in \Omega$. Then $\bar{M} A=\bar{M} \varepsilon$, a direct summand of ${ }_{R} \bar{M}$ and hence closed.

(b) $K=\bar{M} \varepsilon$ for some $\varepsilon^{2}=\varepsilon \in \Omega$, and then $\operatorname{Hom}_{R}(\bar{M}, K)=\Omega \varepsilon$ which is closed.

(c) This follows from the preceding correspondence.

Corollary 7. If $Z\left({ }_{R} M\right)=0$, then $\mathscr{C}\left({ }_{R} M\right)$ is lattice isomorphic to $\mathscr{C}\left({ }_{S} S\right)$.

Proof. $\mathscr{C}\left({ }_{S} S\right) \cong \mathscr{C}\left({ }_{S} \Omega\right) \cong \mathscr{C}\left({ }_{\Omega} \Omega\right)$ by [3, p. 61 and p. 70] and Proposition 4. Hence, by Proposition $6, \mathscr{C}\left({ }_{R} M\right) \cong \mathscr{C}\left({ }_{R} \bar{M}\right) \cong \mathscr{C}\left({ }_{\Omega} \Omega\right) \cong \mathscr{C}\left({ }_{S} S\right)$.

A module ${ }_{R} M$ is atomic if each nonzero element of $\mathscr{C}\left({ }_{R} M\right)$ contains a minimal nonzero element of $\mathscr{C}\left({ }_{R} M\right)$, called an atom. A ring is a full linear ring if it is isomorphic to the full ring of linear transformations of a left vector space over a division ring.

THEOREM 8. S has a maximal left quotient ring which is a direct product of full linear rings if and only if $Z\left({ }_{R} M\right)=0$ and ${ }_{R} M$ is atomic.

Proof. By [6, Theorem 2], $S$ has a maximal left quotient ring which is a direct product of full linear rings if and only if $Z\left({ }_{S} S\right)=0$ and ${ }_{S} S$ is atomic. By virtue of Corollary $7,{ }_{S} S$ is atomic if and only if ${ }_{R} M$ is. The result follows from Proposition 2.

A module ${ }_{R} M$ is $Q$-prime if for any atoms $K_{1}$ and $K_{2}$ of $\mathscr{C}\left({ }_{R} M\right)$ there exist nonzero isomorphic submodules of $K_{1}$ and $K_{2}$ respectively.

Proposition 9. Suppose $Z\left({ }_{R} M\right)=0$ and ${ }_{R} M$ is atomic. Then ${ }_{R} M$ is Q-prime if and only if all atoms of $\mathscr{C}\left({ }_{R} \bar{M}\right)$ are isomorphic; equivalently 
if and only if all atoms of $\mathscr{C}\left({ }_{\Omega} \Omega\right.$ ) are isomorphic. (For the preceding, the standing hypothesis need not hold.) Consequently, ${ }_{R} M$ is $Q$-prime if and only if ${ }_{S} S$ is Q-prime.

Proof. If ${ }_{R} M$ is $Q$-prime and $K_{1}$ and $K_{2}$ are atoms of ${ }_{R} \bar{M}$, then $K_{1} \cap M$ and $K_{2} \cap M$ are atoms of ${ }_{R} M . K_{1} \cap M$ and $K_{2} \cap M$ contain nonzero isomorphic submodules, and so $K_{1} \cong K_{2}$ since $K_{1}$ and $K_{2}$ are injective. Conversely, suppose all atoms of ${ }_{R} \bar{M}$ are isomorphic. If $L_{1}$ and $L_{2}$ are atoms of ${ }_{R} M$, then there exist isomorphic atoms $K_{1}$ and $K_{2}$ of ${ }_{R} \bar{M}$ such that $K_{i} \cap M=L_{i}, i=1,2$. If $f: K_{1} \rightarrow K_{2}$ is an isomorphism, then $X_{1}=$ $L_{2} f^{-1} \cap L_{1}$ and $X_{2}=L_{1} f \cap L_{2}$ are nonzero isomorphic submodules of $L_{1}$ and $L_{2}$. Hence ${ }_{R} M$ is $Q$-prime.

Now suppose that all atoms of ${ }_{R} \bar{M}$ are isomorphic and $A_{1}, A_{2}$ are atoms of $\mathscr{C}\left({ }_{\Omega} \Omega\right)$. By Proposition $6, \bar{M} A_{1}$ and $\bar{M} A_{2}$ are in $\mathscr{C}\left({ }_{R} \bar{M}\right)$. Let $\varphi: \bar{M} A_{1} \rightarrow$ $\bar{M} A_{2}$ be an isomorphism. Define $\theta$ from $A_{1}=\operatorname{Hom}_{R}\left(\bar{M}, \bar{M} A_{1}\right)$ into $A_{2}=$ $\operatorname{Hom}_{R}\left(\bar{M}, \bar{M} A_{2}\right)$ by $\psi \theta=\psi \cdot \varphi$ for $\psi \in A_{1}$. A routine calculation shows that $\theta$ is an isomorphism from $A_{1}$ onto $A_{2}$.

Suppose all atoms of $\Omega$ are isomorphic and let $K_{1}, K_{2}$ be atoms of $\mathscr{C}\left({ }_{R} \bar{M}\right)$. As in Proposition $6, K_{i}=\bar{M} \varepsilon_{i}$ where

$$
\varepsilon_{i}^{2}=\varepsilon_{i} \in \Omega \text { and } \operatorname{Hom}_{R}\left(\bar{M}, K_{i}\right)=\Omega \varepsilon_{i} \in \mathscr{C}\left({ }_{\Omega} \Omega\right)
$$

for $i=1,2$. Since $\Omega \varepsilon_{1} \cong \Omega \varepsilon_{2}$ there exist $\mu, v \in \Omega$ such that $\mu \nu=\varepsilon_{1}$ and $\nu \mu=\varepsilon_{2}$ [7, p. 63]. If $\mu^{\prime}=\left.\mu\right|_{K_{1}}$ and $\nu^{\prime}=\left.v\right|_{K_{2}}$, then $\mu^{\prime} \nu^{\prime}=\mathrm{id}_{K_{1}}$ and $\nu^{\prime} \mu^{\prime}=$ $\operatorname{id}_{K_{2}}$. Thus $K_{1} \cong K_{2}$ and all atoms of ${ }_{R} \bar{M}$ are isomorphic.

THEOREM 10. S has a maximal left quotient ring which is a full linear ring if and only if $Z\left({ }_{R} M\right)=0,{ }_{R} M$ is atomic, and ${ }_{R} M$ is $Q$-prime.

Proof. By previous propositions ${ }_{R} M$ has the above properties if and only if ${ }_{S} S$ does. By [6, Theorem 1] this is the case exactly when $S$ has a full linear ring as its maximal left quotient ring.

THEOREM 11. S has a classical left quotient ring which is simple (semisimple) with minimum condition if and only if $\bar{R}=R / \mathrm{ann}_{R} M$ is prime (semiprime), $m[N, M]=0$ for $m \in M$ implies that $m=0, Z\left({ }_{R} M\right)=0$, and $d\left({ }_{R} M\right)<\infty$.

Proof. By a well-known theorem [4], $S$ has a classical left quotient ring which is simple (semisimple) with minimum condition if and only if $S$ is a prime (semiprime) ring, $Z\left({ }_{S} S\right)=0$, and $d\left({ }_{S} S\right)<\infty$. In view of the earlier propositions, it suffices to prove that $S$ is prime (semiprime) exactly when $\bar{R}$ is prime (semiprime) and $m[N, M]=0$ for $m \in M$ implies that $m=0$.

Suppose that $S$ is prime and let $r, r^{\prime} \in R \backslash$ ann ${ }_{R} M$. Then $r M \neq 0$, $r^{\prime} M \neq 0$, and consequently $[N, r M] \neq 0,\left[N, r^{\prime} M\right] \neq 0$. Since $S$ is prime 
$0 \neq[N, r M]\left[N, r^{\prime} M\right]=\left[N, r(M, N) r^{\prime} M\right] . \quad$ In particular $r(M, N) r^{\prime} M \neq 0$ proving that $\bar{r}(\overline{M, N}) \bar{r}^{\prime} \neq 0$ in $\bar{R}$ ( $\bar{r}$ denotes the coset of $r$ in $\left.\bar{R}\right)$. Hence $\bar{R}$ is prime. Next suppose that $m[N, M]=0$ with $m \in M$. Then $[N, m][N, M]=$ 0 , and since $S$ is prime $[N, m]=0$ proving that $m=0$.

Conversely, suppose that $\bar{R}$ is prime and that $m[N, M]=0$ for $m \in M$ implies that $m=0$. Let $0 \neq s \in S, 0 \neq t \in S$. Then $M s \neq 0$ and $M t \neq 0$ so $M s[N, M] \neq 0$ and $M t[N, M] \neq 0$. Thus $(M s, N) \ddagger$ ann ${ }_{R} M$ and $(M t, N) \ddagger$ ann ${ }_{R} M$. Since $\bar{R}$ is prime $(M s, N)(M t, N)=M(s[N, M] t, N) \ddagger$ ann $_{R} M$. In particular, $s[N, M] t \neq 0$ proving that $S$ is prime.

The semiprime case is obtained by taking $r=r^{\prime}$ and $s=t$ in the above proof.

We remark that $[9$, Theorem 2.3] is a special case of the preceding theorem.

\section{REFERENCES}

1. S. A. Amitsur, Rings of quotients and Morita contexts, J. Algebra 17 (1971), 273298.

2. H. Bass, The Morita theorems, Lecture Notes, University of Oregon, Eugene, Oregon, 1962.

3. C. Faith, Lectures on injective modules and quotient rings, Lecture Notes in Math., no. 49, Springer-Verlag, New York, 1967. MR 37 \#2791.

4. A. W. Goldie, Semi-prime rings with maximum condition, Proc. London Math. Soc. (3) 10 (1960), 201-220. MR 22 \#2627.

5. R. E. Johnson and E. T. Wong, Self-injective rings, Canad. Math. Bull. 2 (1959), 167-173. MR 21 \#5652.

6. J. J. Hutchinson, Quotient full linear rings, Proc. Amer. Math. Soc. 28 (1971), 375-378.

7. J. Lambek, Lectures on rings and modules, Blaisdell, Waltham, Mass., 1966. MR 34 \#5857.

8. F. L. Sandomierski, Semisimple maximal quotient rings, Trans. Amer. Math. Soc. 128 (1967), 112-120. MR 35 \#5473.

9. J. M. Zelmanowitz, Endomorphism rings of torsionless modules, J. Algebra 5 (1967), 325-341. MR 34 \#2626.

Department of Mathematics, Washington State University, Pullman, WashINGTON 99163

Department of Mathematics, University of California, Santa Barbara, CALIFORNIA 93106 